\title{
On Study of Some Concepts in Nano Continuity via Graph Theory
}

\author{
Waleed Ramadhan Khalifa1, Taha Hameed Jasim² \\ ${ }^{1}$ Department of Mathematics, College of Computer Science and Mathematics, Mosul University, Mosul, Iraq \\ ${ }^{2}$ Department of Mathematics, College of Computer Science and Mathematics, Tikrit University, Tikrit, Iraq \\ Email: waleedrmdhan985@gmail.com, tahahameed@tu.edu.iq
}

How to cite this paper: Khalifa, W.R. and Jasim, T.H. (2021) On Study of Some Concepts in Nano Continuity via Graph Theory. Open Access Library Journal, 8: e7568.

https://doi.org/10.4236/oalib.1107568

Received: May 22, 2021

Accepted: July 11, 2021

Published: July 14, 2021

Copyright $\odot 2021$ by author(s) and Open Access Library Inc.

This work is licensed under the Creative Commons Attribution International License (CC BY 4.0).

http://creativecommons.org/licenses/by/4.0/

\section{(c) (i) Open Access}

\begin{abstract}
The purpose of this paper is to introduce a nano-topological space via graph theory which depends on a neighborhood between the vertices based on undirected graph with example. The Concept of Continuity was generalized via graph in order to obtain more of characterization which is application in the graph, we also introduced the concept of nano-dense via graph theory with an example and theorem. At last the concept of homeomorphism was introduced with some examples and theorem.
\end{abstract}

\section{Subject Areas \\ Computer Graphics and Visualization}

\section{Keywords}

Nano-Topology Graph, Nano-Continuity Graph, Nano-Homeomorphism Graph, Nano-Dense Graph

\section{Introduction}

L. Thivagar [1] introduced the concept of nano topological spaces which was defined in terms of approximations and boundary region of a subset of a universe using an equivalence relation on it and also defined nano closed sets, nano interior and nano closure. L. Thivagar [2] introduced a new class of functions on nano-topological spaces called nano continuous functions and derived their characterizations in terms of nano closed sets, nano closure and nano-interior. Atef [3] and Ibrahem [4] studied the relationship between topology and the graph. The two scientists Thivagar [5] and Abd El-Fattah [6] also studied nano-topological via graph theory, where he studied neighborhood between the vertices based on directed graph. In our paper we presented a definition of the 
nano-topological via graph theory, where he studied is neighborhood between the vertices and we studied in our paper an undirected graph theory, also, new class nano continuous function via graph theory.

\section{Preliminaries}

Definition 2.1: [7] [8]

"A graph $G$ is a pair $(V, E)$, where $V$ is nonempty set called vertices or nodes and $E$ is 2-element subsets of $V$ called Edges or links".

Definition 2.2: [7] [8]

"Let $G=(V, E)$ be a graph; we call $H$ a subgraph of $G$ if $V(H) \subseteq V(G)$ and $E(H) \subseteq E(G)$, in which case we write $H \subseteq G$ ". A simple graph $G$ in which each pair of distinct vertices is a complete graph we denote the complete graph on vertices by $k n$ and has $n(n-1) / 2$ edges.

Definition 2.3: [7] [8]

Two graphs $G_{1}$ and $G_{2}$ are isomorphic if there is a one-one correspondence between the vertices of $G_{1}$ and those of $G_{2}$ such that the number of edges joining any two vertices of $G_{1}$ is equal to the number of edges joining the corresponding vertices of $G_{2}$.

\section{Definition 2.4: [1] [9]}

"Let $U$ be a non empty finite set of objects called the universe and $R$ be an equivalence relations on $U$ named as indiscernibility relation. Elements belonging to the same equivalence class are said to be indiscernible with one other. The pair $(U, R)$ is said to be approximation space. Let $X \subseteq U$.

1) The lower approximation of $X$ with respect to $R$ is the set of all objects, which can be certain classified as $X$ with respect to $R$ and is defined by $L_{R}[x]=\bigcup\{R(x): R(X) \subseteq X\}$ where $R(x)$ denotes the equivalence class determined by $X$.

2) The upper approximation of $X$ with respect to $R$ is the set of all objects, which can be possibly classified as $X$ with respect to $R$ and is defined by $U_{R}[V(H)]=\bigcup_{x \in U}\{R(x): R(x) \cap X \neq \varnothing\}$.

3) The boundary region of $X$ with respect to $R$ is the set of all objects, which can be classified as $X$ neither as $X$ nor as not- $X$ with respect to $R$ and is defined by $B_{R}(x)=U_{R}(x)-L_{R}(x)$ ".

\section{Definition 2.5: [1] [9]}

"Let $U$ be the universe and $R$ be an equivalence relation on $U$ and $\tau_{R}(X)=\left\{U, \varnothing, L_{R}(x), U_{R}(x), B_{R}(x)\right\}$. Where $X \subseteq U$, then $\tau_{R}(X)$ satisfies the following axioms:

1) $U$ and $\varnothing \in \tau_{R}(X)$.

2) The union of elements of any sub collection of $\tau_{R}(X)$ is in $\tau_{R}(X)$.

3) The intersection of the elements of any finite sub collection of $\tau_{R}(X)$ is in $\tau_{R}(X)$.

That is $\tau_{R}(X)$ forms a topology on $U$ called as the nano topology on $U$ with respect to $X$. We call $\left(U, \tau_{R}(X)\right)$ as the nano-topological space. The elements 
of $\tau_{R}(X)$ are called as Nano open set".

\section{Definition 2.6: [1] [9]}

"If $\left(U, \tau_{R}(X)\right)$ is a nano topological space with respect to $X$ and if $A \subseteq U$, then the nano interior of $A$ is defined as the union of all nano open subsets of $A$ and it is denoted by $\operatorname{NInt}(A)$ That is, $\operatorname{NInt}(A)$ is the largest nano open subset of $A$. The nano closure of $A$ is defined as the intersection of all nano closed sets containing $A$ and it is denoted by $\operatorname{NCl}(A)$. That is, $\operatorname{NCl}(A)$ is the smallest nano closed set containing $A$ ".

Definition 2.7: [5]

Let $G=(V, E)$ be a graph, $v \in V(G)$. Then the neighborhood of $v$ and denote that $\mathrm{N}(v)$ weredefined by $\mathrm{N}(v)=\{v\} \cup\{u \in V(G): \overline{v u} \in E(G)\}$.

Definition 2.8: [5]

Let $G=(V, E)$ be a graph, $H$ be a subgraph from $G, \mathrm{~N}(v)$ is neighborhood of $v$ in $V(G)$; Then

1) The lower approximation $L: P(V(G)) \rightarrow P(V(G))$ is

$$
L_{N}[V(H)]=\bigcup_{v \in V(G)}\{v: \mathrm{N}(v) \subseteq V(H)\} .
$$

2) The upper approximation $U: P(V(G)) \rightarrow P(V(G))$ is

$$
U_{N}[V(H)]=\bigcup_{v \in V(G)}\{N(v): \mathrm{N}(v) \cap V(H) \neq \varnothing\} .
$$

3) The boundary region is $B_{N}[V(H)]=U_{N}[V(H)]-L_{N}[V(H)]$.

\section{Nano Topological Space via Graph Theory}

In this section we have studied the concept of nano topological space via graph theory with example.

\section{Definition 3.1:}

"Let $G=(V, E), \mathrm{N}(v)$ is neighborhood of $v$ in $V, H$ be a subgraph from $G$ $\tau_{N}[V(H)]=\left\{V(G), \varnothing, L_{N}[V(H)], U_{N}[V(H)], B_{N}[V(H)]\right\}$. Forms a topology on $V(G)$ called nano-topology on $V(G)$ with respect to $V(H)$.

$\left(V(G), \tau_{N}[V(H)]\right)$ is called nano-topological graph".

\section{Example 3.2:}

"Let $G=(V, E)$ be a graph (see Figure 1). Then, $\mathrm{N}\left(v_{1}\right)=\left\{v_{1}, v_{4}\right\}$, $\mathrm{N}\left(v_{2}\right)=\left\{v_{2}, v_{3}\right\}, \mathrm{N}\left(v_{3}\right)=\left\{v_{2}, v_{3}, v_{4}\right\}, \mathrm{N}\left(v_{4}\right)=\left\{v_{1}, v_{3}, v_{4}\right\} . H$ is a subgraph with vertices $V(H)=\left\{v_{2}, v_{3}\right\}$, then $L_{N}[V(H)]=\left\{v_{1}\right\}, U_{N}[V(H)]=\left\{v_{1}, v_{3}, v_{4}\right\}$, $B_{N}[V(H)]=\left\{v_{3}, v_{4}\right\}$. Therefore, the nano-topology from $G$ will be $\tau_{N}[V(H)]=\left\{V(G), \varnothing,\left\{v_{2}\right\},\left\{v_{2}, v_{3}, v_{4}\right\},\left\{v_{3}, v_{4}\right\}\right\}$.

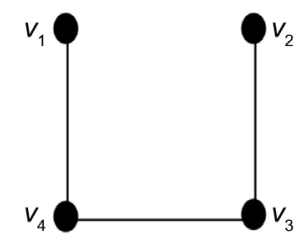

Figure 1. Simple graph (nano-topology test of connected graph without cycle). 


\section{Nano Continuity via Graph Theory}

In this section we studied the concept nano continuity via graph theory with some characterization, examples and proofs.

\section{Definition 4.1:}

Let $G=(V, E)$ and $G_{1}=\left(V_{1}, E_{1}\right)$ be any two isomorphic graphs with nano topological graph $\left(V(G), \tau_{N}(V(H))\right),\left(V\left(G_{1}\right), \tau_{N}(f(V(H)))\right)$. Then the mapping $f:\left(V(G), \tau_{N}(V(H))\right) \rightarrow\left(V\left(G_{1}\right), \tau_{N}(f(V(H)))\right)$ is called nano-continuous on $V\left(G_{1}\right)$ if the inverse image from each nano-open set in $V(G)$ is nano-open in $V\left(G_{1}\right)$.

\section{Example 4.2:}

Let $G_{1}=\left(V_{1}, E_{1}\right), G_{2}=\left(V_{2}, E_{2}\right)$ be two isomorphic graphs (see Figure 2). Then there exists a function $f: V\left(G_{1}\right) \rightarrow V\left(G_{2}\right)$ as $f\left(v_{1}\right)=u_{2}, f\left(v_{2}\right)=u_{3}$, $f\left(v_{3}\right)=u_{4}, f\left(v_{4}\right)=u_{1}$. We a construct nano-topology on $G_{1}$. Assume that $H$ is a subgraph from $G_{1}$ with vertices $V(H)=\left\{v_{1}, v_{2}, v_{4}\right\}$.

Then $\mathrm{N}\left(v_{1}\right)=\left\{v_{1}, v_{2}, v_{4}\right\}, \mathrm{N}\left(v_{2}\right)=V(G), \mathrm{N}\left(v_{3}\right)=\left\{v_{2}, v_{3}, v_{4}\right\}, \mathrm{N}\left(v_{4}\right)=V(G)$. Since, $L_{N}[V(H)]=\left\{v_{1}\right\}, U_{N}[V(H)]=V(G), B_{N}[V(H)]=\left\{v_{2}, v_{3}, v_{4}\right\}$. Then the nano-topological graph from $V(H)$ are

$\tau_{N}(V(H))=\left\{V\left(G_{1}\right), \varnothing,\left\{v_{1}\right\},\left\{v_{2}, v_{3}, v_{4}\right\}\right\}$.

We a construct nano-topology on $G_{2}$ generated by $f(V(H))=\left\{u_{1}, u_{2}, u_{3}\right\}$.

Assume that $W$ is a subgraph from $G_{2}$ with vertices $V(W)=\left\{u_{2}, u_{4}\right\}$. Then, $\mathrm{N}\left(u_{1}\right)=V(G), \mathrm{N}\left(u_{2}\right)=\left\{u_{1}, u_{2}, u_{3}\right\}, \mathrm{N}\left(u_{3}\right)=V(G), \mathrm{N}\left(u_{4}\right)=\left\{u_{1}, u_{3}, u_{4}\right\}$. Since, $L_{N}[V(H)]=\left\{u_{2}\right\}, U_{N}[V(H)]=V(G), B_{N}[V(H)]=\left\{u_{1}, u_{3}, u_{4}\right\}$. Then, the nano-topological graph from $f(V(H))$ are

$\tau_{N}(f(V(H)))=\left\{V\left(G_{2}\right), \varnothing,\left\{u_{2}\right\},\left\{u_{1}, u_{3}, u_{4}\right\}\right\}$. Then, $f^{-1}\left\{u_{1}, u_{3}, u_{4}\right\}=\left\{v_{2}, v_{3}, v_{4}\right\}$, $f^{-1}\left\{u_{2}\right\}=\left\{v_{1}\right\}$ Therefore, $f$ is nano continuous.

\section{Theorem 4.3:}

"Let $G=(V, E)$ and $G_{1}=\left(V_{1}, E_{1}\right)$ be any two isomorphic graphs with nano topological graph $\left(V(G), \tau_{N}(V(H))\right),\left(V\left(G_{1}\right), \tau_{N}(f(V(H)))\right)$.

A function $f:\left(V(G), \tau_{N}(V(H))\right) \rightarrow\left(V\left(G_{1}\right), \tau_{N}(f(V(H)))\right)$ is nano continuous if and only if the inverse image of every nano closed set in $V\left(G_{1}\right)$ is nano closed in $V(G)$ ".

\section{Proof:}

"Let $f$ be nano continuous and $V(F)$ be nano closed in $V\left(G_{1}\right)$. That is, $V\left(G_{1}\right)-V(F)$ is nano-open in $V\left(G_{1}\right)$. Since $f$ is nano continuous, $f^{-1}\left(V\left(G_{1}\right)-V(F)\right)$ is nano-open in $V(G)$. That is, $V(G)-f^{-1}(V(F))$ is
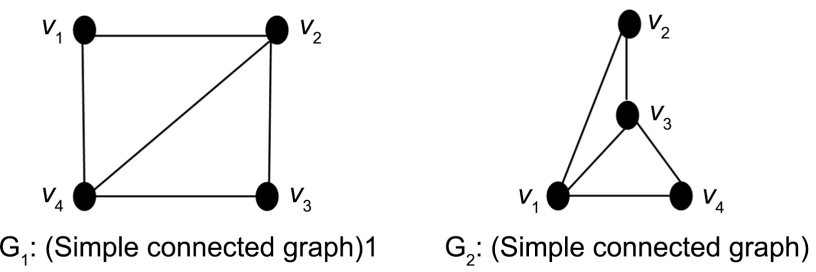

Figure 2. Two isomorphic graphs (the continuity test of nano topological space via graph theory). 
nano-open in $V(G)$. Therefore, $f^{-1}(V(F))$ is nano closed in $V(G)$. Thus, the inverse image of every nano closed set in $V\left(G_{1}\right)$ is nano closed in, if $f$ is nano continuous on $V(G)$. Conversely, let the inverse image of every nano closed set be nano closed. Let $V(H)$ be nano-open in $V\left(G_{1}\right)$. Then $V\left(G_{1}\right)-V(H)$ is nano closed in $V\left(G_{1}\right)$. Then, $f^{-1}\left(V\left(G_{1}\right)-V(H)\right)$ is nano closed in $V(G)$. That is, $V(G)-f^{-1}(V(H))$ is nano closed in $V(G)$. Therefore, $f^{-1}(V(H))$ is nano-open in $V(G)$. Thus, the inverse image of every nano-open set in $V\left(G_{1}\right)$ is nano-open in $V(G)$. That is, $f$ is nano continuous on $V(G)$ ".

\section{Theorem 4.4:}

Let $G=(V, E)$ and $G_{1}=\left(V_{1}, E_{1}\right)$ be any two isomorphic graphs with nano topological graph $\left(V(G), \tau_{N}(V(H))\right),\left(V\left(G_{1}\right), \tau_{N}(f(V(H)))\right)$. A function $f:\left(V(G), \tau_{N}(V(H))\right) \rightarrow\left(V\left(G_{1}\right), \tau_{N}(f(V(H)))\right)$ is nano continuous if and only if $f\left(N_{G}(V(H))\right) \subseteq N C l_{G}(f(V(H)))$ for every subgraph $H$ of $G$.

\section{Proof:}

"Let $f$ be nano continuous and $V(H) \subseteq V(G)$. Then $f(V(H)) \subseteq V\left(G_{1}\right)$. $N_{G}(f(V(H)))$ is nano closed in $V\left(G_{1}\right)$. Since $f$ is nano continuous, $f^{-1}\left(N_{C l}(f(V(H)))\right)$ is nano closed in $V(G)$. Since

$f(V(H)) \subseteq N C l_{G}(f(V(H))), V(H) \subseteq f^{-1}\left(N C l_{G}(f(V(H)))\right)$. Thus $f^{-1}\left(\mathrm{NCl}_{G}(f(V(H)))\right)$ is a nano closed set containing $V(H)$. But, $N_{C l}(V(H))$ is the smallest nano closed set containing $V(H)$. Therefore,

$N C l_{G}(V(H)) \subseteq f^{-1}\left(N_{C l}(f(V(H)))\right)$. That is, $f\left(N_{G}(V(H))\right) \subseteq N C l_{G}(f(V(H)))$.

Conversely, let $f\left(N_{C l}(V(H))\right) \subseteq N C l_{G}(f(V(H)))$ for every subgraph $V(H)$ of $V(G)$. If $V(F)$ is nano closed in $V\left(G_{1}\right)$, since $f^{-1}(V(F)) \subseteq V(G)$, $f\left(N_{G} l_{G}\left(f^{-1}(V(F))\right)\right) \subseteq N C l_{G}\left(f\left(f^{-1}(V(F))\right)\right) \subseteq N C l_{G}(V(F))$.

That is, $C l_{G}\left(f^{-1}(V(F))\right) \subseteq f^{-1}\left(N C l_{G}(V(F))\right)=f^{-1}(V(F))$, since $V(F)$ is nano closed. Thus $N C l_{G}\left(f^{-1}(V(F))\right) \subseteq f^{-1}(V(F))$ But,

$f^{-1}(V(F)) \subseteq N C l_{G}\left(f^{-1}(V(F))\right)$. Therefore, $N C l_{G}\left(f^{-1}(V(F))\right)=f^{-1}(V(F))$. Therefore, $f^{-1}(V(F))$ is nano closed in $V(G)$ for every nano closed set $V(F)$ in $V\left(G_{1}\right)$. That is, $f$ is nano continuous".

\section{Theorem 4.5:}

"Let $G=(V, E)$ and $G_{1}=\left(V_{1}, E_{1}\right)$ be any two isomorphic graphs with nano topological graph $\left(V(G), \tau_{N}(V(H))\right),\left(V\left(G_{1}\right), \tau_{N}(f(V(H)))\right)$. A function $f:\left(V(G), \tau_{N}(V(H))\right) \rightarrow\left(V\left(G_{1}\right), \tau_{N}(f(V(H)))\right)$ is nano continuous if and only if $N_{C l}\left(f^{-1}(V(H))\right) \subseteq f^{-1}\left(N C l_{G}(V(H))\right)$ for every subgraph $H$ of $G$ '.

\section{Proof:}

"If $f$ is nano continuous and $V(H) \subseteq V\left(G_{1}\right), \quad N C l_{G}(V(H))$ is nano closed in $V\left(G_{1}\right)$ and hence $f^{-1}\left(N_{C l}(V(H))\right)$ is nano closed in $V(G)$. Therefore, $N_{C l}\left(f^{-1}\left(N C l_{G}(V(H))\right)\right)=f^{-1}\left(N C l_{G}(V(H))\right)$. Since $V(H) \subseteq N C l_{G}(V(H)) \Rightarrow f^{-1}(V(H)) \subseteq f^{-1}\left(N C l_{G}(V(H))\right)$. Therefore, $N C l_{G}\left(f^{-1}(V(H))\right) \subseteq N C l_{G}\left(f^{-1}\left(N C l_{G}(V(H))\right)\right)=f^{-1}\left(N C l_{G}(V(H))\right)$. That is, $N_{C l}\left(f^{-1}(V(H))\right) \subseteq f^{-1}\left(N_{G}(V(H))\right)$. 
Conversely, assume that $N_{C l}\left(f^{-1}(V(H))\right) \subseteq f^{-1}\left(N C l_{G}(V(H))\right)$ for every $V(H) \subseteq V\left(G_{1}\right)$. Let $V(H)$ be nano closed in $V\left(G_{1}\right)$. Then $N_{C l}(V(H))=V(H)$. By Assumption,

$N_{C l}\left(f^{-1}(V(H))\right) \subseteq f^{-1}\left(N C l_{G}(V(H))\right)=f^{-1}(V(H))$. Thus, $N C l_{G}\left(f^{-1}(V(H))\right) \subseteq f^{-1}(V(H))$. But, $f^{-1}(V(H)) \subseteq N C l_{G}\left(f^{-1}(V(H))\right)$. Therefore, ${ }_{N C l}\left(f^{-1}(V(H))\right)=f^{-1}(V(H))$. That is, $f^{-1}(V(H))$ is nano closed in $V(G)$ for every nano closed set $V(H)$ in $V\left(G_{1}\right)$. Therefore, $f$ is nano continuous on $V(G)$ ".

\section{Theorem 4.6:}

Let $G=(V, E)$ and $G_{1}=\left(V_{1}, E_{1}\right)$ be any two isomorphic graphs with nano topological graph $\left(V(G), \tau_{N}(V(H))\right),\left(V\left(G_{1}\right), \tau_{N}(f(V(H)))\right)$. A function $f:\left(V(G), \tau_{N}(V(H))\right) \rightarrow\left(V\left(G_{1}\right), \tau_{N}(f(V(H)))\right)$ is nano continuous on $V(G)$ if and only if $f^{-1}\left(\operatorname{NInt}_{G}(V(H))\right) \subseteq N \operatorname{NIt}_{G}\left(f^{-1}(V(H))\right)$ for every subgraph $H$ of $G_{1}$.

\section{Proof:}

Assume that $f$ is nano continuous and $V(H) \subseteq V\left(G_{1}\right), \operatorname{NInt}_{G}(V(H))$ is nano open in $\left(V\left(G_{1}\right), \tau_{N}(f(V(H)))\right)$ and hence $f^{-1}\left(\operatorname{NInt}_{G}(V(H))\right)$ is nano open in $\left(V(G), \tau_{N}(V(H))\right)$. Therefore, $f^{-1}\left(\operatorname{NInt}_{G}(V(H))\right)=\operatorname{NInt}_{G}\left(f^{-1}\left(\operatorname{NInt}_{G}(V(H))\right)\right)$. Since $\operatorname{NInt}_{G}(V(H)) \subseteq V(H) \Rightarrow f^{-1}\left(\operatorname{NInt}_{G}(V(H))\right) \subseteq f^{-1}(V(H))$. Therefore, $\operatorname{NInt}_{G}\left(f^{-1}\left(\operatorname{NInt}_{G}(V(H))\right)\right) \subseteq \operatorname{NInt}_{G}\left(f^{-1}(V(H))\right)=f^{-1}\left(N C l_{G}(V(H))\right)$. That is $f^{-1}\left(\operatorname{NInt}_{G}(V(H))\right) \subseteq N \operatorname{NInt}_{G}\left(f^{-1}(V(H))\right)$.

Conversely, assume that $f^{-1}\left(\operatorname{NInt}_{G}(V(H))\right) \subseteq N \operatorname{Nin}_{G}\left(f^{-1}(V(H))\right)$ for every $V(H) \subseteq V\left(G_{1}\right)$. If $V(H)$ be nano open in $V\left(G_{1}\right)$. $\operatorname{NInt}_{G}(V(H))=V(H)$. By assumption, $\operatorname{NInt}_{G}\left(f^{-1}(V(H))\right) \subseteq \operatorname{NInt}_{G}\left(f^{-1}(V(H))\right)$. That is, $f^{-1}(V(H)) \subseteq \operatorname{NInt}_{G}\left(f^{-1}(V(H))\right)$. But, $\operatorname{NInt}_{G}\left(f^{-1}(V(H))\right) \subseteq f^{-1}(V(H))$. Therefore, $f^{-1}(V(H))=\operatorname{NInt}_{G}\left(f^{-1}(V(H))\right)$. That is, $f^{-1}(V(H))$ is nano open in $V(G)$ for every nano open set $V(H)$ in $V\left(G_{1}\right)$. Therefore, $f$ is nano continuous.

\section{Definition 4.7:}

Let $G=(V, E)$ be a graph with nano-topological graph $\left(V(G), \tau_{N}(V(H))\right)$. If $W \subseteq G$ is said to be nano-dense if $N_{C l}(V(W))=V(G)$.

\section{Example 4.8:}

From example 4.2. Assume that $H$ is a subgraph from $G_{1}$ with vertices $V(H)=\left\{v_{1}, v_{2}, v_{4}\right\}$. Then $\mathrm{N}\left(v_{1}\right)=\left\{v_{1}, v_{2}, v_{4}\right\}, \mathrm{N}\left(v_{2}\right)=V(G), \mathrm{N}\left(v_{3}\right)=\left\{v_{2}, v_{3}, v_{4}\right\}$, $\mathrm{N}\left(v_{4}\right)=V(G)$. Since, $C\left(\mathrm{~N}\left(v_{1}\right)\right)=\left\{v_{3}\right\}, C\left(\mathrm{~N}\left(v_{2}\right)\right)=\varnothing, C\left(\mathrm{~N}\left(v_{3}\right)\right)=\left\{v_{1}\right\}, C($ $\left.\mathrm{N}\left(v_{4}\right)\right)=\varnothing$. Then, $C\left(\mathrm{~N}\left(v_{3}\right)\right) \cap V(H) \neq \varnothing$. Therefore, $N C l_{G}(V(H))=U_{N}[V(H)] \bigcup\left\{v_{3}\right\}=V\left(G_{1}\right)$. Hence $N_{C l}(V(H))=V\left(G_{1}\right)$.

\section{Theorem 4.9:}

Let $G=(V, E)$ and $G_{1}=\left(V_{1}, E_{1}\right)$ be any two isomorphic graphs with nano topological graph $\left(V(G), \tau_{N}(V(H))\right),\left(V\left(G_{1}\right), \tau_{N}(f(V(H)))\right)$ and $f:\left(V(G), \tau_{N}(V(H))\right) \rightarrow\left(V\left(G_{1}\right), \tau_{N}(f(V(H)))\right)$ be an onto and nano continuous function, $V(H) \subseteq V(G)$. If $V(H)$ is nano dense in $V(G)$, then 
$f(V(H))$ is nano dense in $V\left(G_{1}\right)$.

\section{Proof:}

"Since $V(H)$ is nano dense in $V(G), N_{G}(V(H)) \subseteq V(G)$. Then $f\left(N_{G}(V(H))\right) \subseteq f(V(G))=V\left(G_{1}\right)$, since $f$ is onto and nano continuous on $V(G), f\left(N_{G}(V(H))\right) \subseteq N C l_{G}(f(V(H)))$. Therefore, $V\left(G_{1}\right) \subseteq N C l_{G}(V(H))$ but $N C l_{G}(V(H)) \subseteq V\left(G_{1}\right)$. Therefore, $N C l_{G}(V(H))=V\left(G_{1}\right)$. That is $f(V(H))$ is dense in $V\left(G_{1}\right)$. Thus, a nano continuous function maps nano dense sets into nano dense sets, provided it is onto".

\section{Nano Homeomrphism via Graph Theory}

In this section we studied of nano homeomorphism in the graph with some examples and proofs.

\section{Definition 5.1:}

Let $G=(V, E)$ and $G_{1}=\left(V_{1}, E_{1}\right)$ be any two isomorphic graphs with nano topological graph $\left(V(G), \tau_{N}(V(H))\right),\left(V\left(G_{1}\right), \tau_{N}(f(V(H)))\right)$. A function $f:\left(V(G), \tau_{N}(V(H))\right) \rightarrow\left(V\left(G_{1}\right), \tau_{N}(f(V(H)))\right)$ is nano-open map if the image of every nano-open set in $V(G)$ is nano open in $V\left(G_{1}\right)$. The mapping $f$ is said to be a nano closed set map if the image of every nano-closed set in $V(G)$ is nano closed in $V\left(G_{1}\right)$.

Theorem 5.2:

Let $G=(V, E)$ and $G_{1}=\left(V_{1}, E_{1}\right)$ be any two isomorphic graphs with nano topological graph $\left(V(G), \tau_{N}(V(H))\right),\left(V\left(G_{1}\right), \tau_{N}(f(V(H)))\right)$ A mapping $f:\left(V(G), \tau_{N}(V(H))\right) \rightarrow\left(V\left(G_{1}\right), \tau_{N}(f(V(H)))\right)$ is nano closed map if and only if $N_{G}(f(V(H))) \subseteq f\left(N C l_{G}(V(H))\right)$, for every subgraph $H \subseteq G$.

\section{Proof:}

If $f$ is nano closed, $f\left(N_{C}(V(H))\right)$ is nano closed in $V\left(G_{1}\right)$, since $N C l_{G}(V(F))$ is nano closed $V(G)$. Then, $V(H) \subseteq N C l_{G}(V(H))$, $f(V(H)) \subseteq f\left(N_{G}(V(H))\right)$. Thus, $f\left(N C l_{G}(V(H))\right)$ is a nano closed set containing $f(V(H))$. Therefore, $\operatorname{NCl}_{G}(f(V(H))) \subseteq f\left(N_{C l}(V(H))\right)$.

Conversely, if $V(F)$ is nano closed in $V(G)$, then $N_{C l}(V(F))=V(F)$ and hence $f(V(F)) \subseteq N C l_{G}(f(V(F))) \subseteq f\left(N_{G}(V(F))\right)=f(V(F))$. Thus, $V(F)=N C l_{G}(V(F))$. That is, $f(V(F))$ is nano closed in $V\left(G_{1}\right)$. Therefore, $f$ is nano closed map.

\section{Theorem 5.3:}

Let $G=(V, E)$ and $G_{1}=\left(V_{1}, E_{1}\right)$ be any two isomorphic graphs with nano topological graph $\left(V(G), \tau_{N}(V(H))\right),\left(V\left(G_{1}\right), \tau_{N}(f(V(H)))\right)$ A mapping $f:\left(V(G), \tau_{N}(V(H))\right) \rightarrow\left(V\left(G_{1}\right), \tau_{N}(f(V(H)))\right)$ is nano open map if and only if $f\left(N_{G} t_{G}(V(H))\right) \subseteq \operatorname{NInt}_{G}(f(V(H)))$, for every subgraph $H \subseteq G$.

Proof: It's clear by theorem

\section{Definition 5.4:}

Let $G=(V, E)$ and $G_{1}=\left(V_{1}, E_{1}\right)$ be any two isomorphic graphs with nano topological graph $\left(V(G), \tau_{N}(V(H))\right)$. Then the mapping 
$f:\left(V(G), \tau_{N}(V(H))\right) \rightarrow\left(V\left(G_{1}\right), \tau_{N}(f(V(H)))\right)$ is called nano-homeomorphism if

1) $f$ is one to one and onto.

2) $f$ is nano-continuous.

3) $f$ is nano-open.

\section{Example 5.5:}

From Example 4.2. Assume that $H$ is a subgraph from $G$ with vertices $V(H)=\left\{v_{1}, v_{2}, v_{4}\right\}$. Then the nano-topological graph from $V(H)$ and $f(V(H))$ are $\tau_{N}(V(H))=\left\{V\left(G_{1}\right), \varnothing,\left\{v_{1}\right\},\left\{v_{2}, v_{3}, v_{4}\right\}\right\}$. $\tau_{N}(f(V(H)))=\left\{V\left(G_{2}\right), \varnothing,\left\{u_{2}\right\},\left\{u_{1}, u_{3}, u_{4}\right\}\right\}$. Then the function $f:\left(V\left(G_{1}\right), \tau_{N}(V(H))\right) \rightarrow\left(V\left(G_{2}\right), \tau_{N}(f(V(H)))\right)$ is nano-homeomorphism.

\section{Theorem 5.6:}

"Let $G=(V, E)$ and $G_{1}=\left(V_{1}, E_{1}\right)$ be any two isomorphic graphs with nano topological graph $\left(V(G), \tau_{N}(V(H))\right)$ and $f:\left(V(G), \tau_{N}(V(H))\right) \rightarrow\left(V\left(G_{1}\right), \tau_{N}(f(V(H)))\right)$ is one-one and onto. Then $f$ is a nano homeomorphism if and only if $f$ is nano closed and nano continuous".

\section{Proof:}

"Let $f$ is a nano homeomorphism. Then $f$ is nono continuous. Let $V(F)$ be an arbitrary nano closed set in $\left(V(G), \tau_{N}(V(H))\right)$. Then $V(G)-V(F)$ is nano open, since $f$ is nano open, $f(V(G)-V(F))$ is nano open $V\left(G_{1}\right)$. That is, $V\left(G_{1}\right)-f(V(F))$ is nano open in $V\left(G_{1}\right)$. Therefore, $f(V(F))$ is nano closed in $V\left(G_{1}\right)$. Thus, the image of every nano closed set in $V(G)$ is nano closed in $V\left(G_{1}\right)$. That is $f$ is nano closed.

Conversely, let $f$ is nano closed and continuous. Let $V(H)$ be a nano open set in $\left(V(G), \tau_{N}(V(H))\right)$. Then $V(G)-V(H)$ is nano closed in $V(G)$, since $f$ is nano closed, $f(V(G)-V(H))=V\left(G_{1}\right)-f(V(H))$ is nano closed in $V\left(G_{1}\right)$. Therefore, $f(V(F))$ is nano open in $V\left(G_{1}\right)$. Thus, $f$ is nano open and hence $f$ is a nano homeomorphism".

\section{Theorem 5.7:}

Let $G=(V, E), G_{1}=\left(V_{1}, E_{1}\right)$ with nano topological graph $\left(V(G), \tau_{N}(V(H))\right)$. A one-one $f$ of $\left(V(G), \tau_{N}(V(H))\right)$ onto $\left(V\left(G_{1}\right), \tau_{N}(f(V(H)))\right)$ is a nano homeomorphism iff $f\left(N C l_{G}(V(H))\right) \subseteq N C l_{G}(f(V(H)))$ for every subgraph $H$ of $G$.

\section{Proof:}

If $f$ is a nano homeomorphism, $f$ is nano continuous and nano closed. If $V(H) \subseteq V(G), \quad f\left(N_{G}(V(H))\right) \subset N C l_{G}(f(V(H)))$, since $f$ is nano continuous. Then ${ }^{N C l}(V(H))$ is nano closed in $V(H)$ and $f$ is nano closed, $f\left(N_{G}(V(H))\right)$ is nano closed in $V\left(G_{1}\right)$.

$N C l_{G}\left(f\left(N_{G}(V(H))\right)\right)=f\left(N C l_{G}(V(H))\right)$. Since $V(H) \subseteq N C l_{G}(V(H))$, $f(V(H)) \subseteq f\left(N_{G}(V(H))\right)$ and hence

$N_{C l}(f(V(H))) \subseteq N C l_{G}\left(f\left(N C l_{G}(V(H))\right)\right)=f\left(N C l_{G}(V(H))\right)$. Therefore, $N C l_{G}(f(V(H))) \subseteq f\left(N C l_{G}(V(H))\right)$. Thus, 
$f\left(N_{G}(V(H))\right)=N C l_{G}(f(V(H)))$ if $f$ a nano homeomorphism.

Conversely, if $V(H)$ is nano-closed in $V(G) \cdot N_{G}(V(H))=V(H)$ which implies $f\left(N_{C l}(V(H))\right)=f(V(H))$. Therefore, $N_{C l}(f(V(H)))=f(V(H))$, thus $V(H)$ is nano closed in $V\left(G_{1}\right)$, for every nano closed set $V(H)$ in $V(G)$. That is $f$ is nano closed. Also $f$ is nano continuous. Thus, $f$ is a nano homeomorphism.

\section{Conclusion}

In this paper, the relationship between nano topology and graph theory was studied to show how nano topology is deduced from any graph as explained in Section 3. We also deduced the concepts of continuity and homeomorphism in nano topology and their relationship with two isomorphic graphs as explained in Section 4 and Section 5.

\section{Conflicts of Interest}

The authors declare no conflicts of interest.

\section{References}

[1] Thivagar, M.L. and Richard, C. (2013) On Nano Forms of Weakly Open Sets. International Journal of Mathematics and Statistics Invention, 1, 31-37.

[2] Thivagar, M.L. and Richard, C. (2013) On Nano Continuity, Mathematical Theory and Modeling, 3, 32-37.

[3] Atef, M. (2018) Some Topological Applications on Graph Theory and Information Systems'. Master thesis, University of Menoufia, Egypt.

[4] Ibrahem, A. (2020) On Topological Graph Theory. Master Thesis, University of Mosul, Iraq.

[5] Lellis Thivagar, M., Manuel, P. and Sutha Devi, V. (2016) Adetection for Patent Infringement Suit via Nanotopology Induced by Graph. Cogent Mathematics, 3, 1-10. https://doi.org/10.1080/23311835.2016.1161129

[6] Nasef, A. and El-Atik, A.F. (2017) Some Properties on Nano Topology Induced by Graphs. AASCIT Journal of Nanoscience, 3, 19-23.

[7] Chartrand, G., Lesniak, L. and Zhang, P. (2016) Textbooks in Mathematics "Graphs and Digraphs"'. Taylor and Francis Group, LLC, California.

[8] Diestel, R. (2000 'Graph Theory. Springer-Verlag Heidelberg Press, New York.

[9] Thivagar, M.L. and Richard, C. (2017) Note on Nano Topological Spaces. (Communicated) 